\title{
Small and Mid-Size Pump-Turbines with Variable Speed
}

\author{
Jürgen Krenn, Helmut Keck, Manfred Sallaberger \\ Andritz Hydro Ltd., Zurich, Switzerland \\ Email: contact-hydro@andritz.com
}

Received February 6, 2013; revised March 8, 2013; accepted March 22, 2013

Copyright (c) 2013 Jürgen Krenn et al. This is an open access article distributed under the Creative Commons Attribution License, which permits unrestricted use, distribution, and reproduction in any medium, provided the original work is properly cited.

\begin{abstract}
The stability of the grid is jeopardized with the large percentage of non-dispatchable renewables like wind power and also with increasing solar power. This creates various problems because these forms of energy are very volatile and difficult to predict. In most countries the in-feed of these sources must not be curtailed. In addition most of the renewables do not provide short circuit capacity and inertia in the same way as classical units and so further worsen the stability of the grid. The growing exploitation of wind and solar might be limited due to grid stability problems. In order to compensate those problems a large amount of reserve capacity is needed and therefore new technologies for electricity storage are required. Hydraulic pumped storage—the classical storage technology —has some disadvantages. These plants are in mountain regions often far away from wind farms. The distance to the wind farms mean additional loading for the already stressed grid and additional transmission losses. To compensate the very volatile wind energy, the pump input power should be varied continuously. This is so far only possible with variable speed units. Up to now double-fed asynchronous motor-generators are used which are rather expensive. In order to provide a solution for the described situation, ANDRITZ HYDRO has developed a new innovative concept of decentralized pump storage plants. Small standardized pump turbines are combined with a synchronous motor-generator and a full size converter which allows speed variation in pump and turbine mode over a wide range. These plants can be built locally close to wind farms and other sources to be balanced, allowing the increase of renewable energy without increasing the transmission line capacity. For the future smart grids this will be a key storage technology. This concept is reliable, innovative and more economic than other storage technologies.
\end{abstract}

Keywords: Grid Stability; Pump Storage; Variable Speed; Full Size Converter; Wind Energy

\section{Introduction}

The main topics of the current discussions about the change in the energy market are well-known. It is about liberalisation, separation of production-transmissiondistribution, increased application of renewable energy and technologies free of carbon dioxide, visions of electro mobility and smart grid [1].

All over the world we face a growing public scepticism against the construction of large scale plants and the installation of new transmission lines. So the need for decentralized energy production is widespread, with fast growing wind and solar energy generation. The following questions arise: how can today's grid infrastructure cope with this? Which type of energy storage method is required in the future? In that context which role plays hydro power and especially pumped storage?

The history of variable speed pump storage plants dates back to the 1990s. At this time several units have been put into operation by Hitachi and Toshiba in Japan.
In Europe two references are available, one for the plant Avce, Slovenia installed by Hitachi and one for the plant Goldisthal, Germany, installed by Andritz Hydro (formerly VA TECH Escher Wyss).

Goldisthal is one of the biggest pump storage power plants in Europe and is equipped with four pump turbines, two with constant speed and two with variable speed [2]. The pump turbines Goldisthal demonstrate with their modern optimized design and the variable speed feasibility that operation behaviour under different heads can be extremely improved. Mechanical design together with the civil structure has been optimised and not only the variable speed but also the fixed speed units show excellent operation behaviour [3].

The continuously growing demand for operating flexibility [4] has led to the current upswing for variable speed pumped storage in Europe. Therefore several plants are under installation in Switzerland and Portugal with planned commissioning in the next years. 


\section{Demand of Energy Storage}

The percentage of renewable energy in the electricity portfolio is increasing all over the world. This creates various problems because the wind and solar energy is very volatile (see Figure 1) and difficult to predict and it is permitted to curtail these in-feeds. If the weather conditions are good then energy is produced and it has to be taken by "the grid", thus in reality by the consumer. Therefore the stability of the grid is in danger with large percentage of non-dispatchable wind power and also with increasing solar power. In all cases the transmission system operator has to ensure that the grid stability is still maintained.

The further exploitation of wind might be limited due to grid stability issues. In order to handle this situation a large amount of reserve capacity is needed and therefore new technologies for electricity storage are required.

The classical storage technology is hydraulic pumped storage. However, large hydraulic pumped storage plants are in mountain regions often far away from wind farms. The distance between these plants and the wind farms mean additional loading for the already stressed grid and additional transmission losses. To compensate the very volatile wind energy, the pump input power should be varied continuously. This was so far only possible with variable speed units (double-fed asynchronous motorgenerators). These units are rather expensive and are often designed as large scale plants. Therefore the public acceptance to realize them is low and the approval process to build may take long and is difficult.

\section{The New Concept of Small and Mid-Size Pump-Turbines}

In order to provide a solution for above mentioned problems, ANDRITZ HYDRO has developed a new innovative concept. In order to establish deeper understanding of that concept, first some insights in the heart of the hydraulic machine are presented.

\subsection{Hydraulic Layout of Pump-Turbines}

To determine the size of a pump-turbine some basic input data is required. Variation of the upper and lower reservoir level, flow or power and the head losses are essential in order to start the layout.

The hydraulic layout of a pump-turbine is done as a pump. The maximum pump net head is determined by adding the head losses to the static head (see Figure 2) while in turbine operation the head losses are subtracted from the static head. Consequently the maximum net head in pump mode is larger than the maximum net head in turbine mode. Therefore maximum net head in pump mode is decisive for the hydraulic layout of the machine and determines the size of the pump outlet diameter of

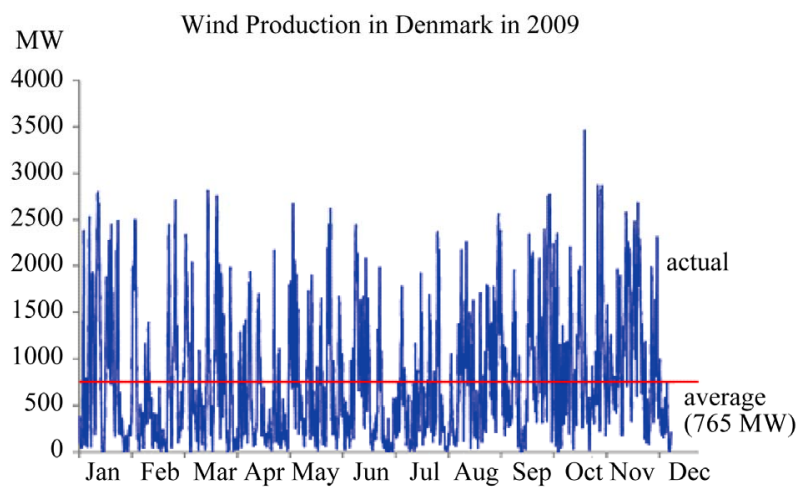

Figure 1. Example for wind production.

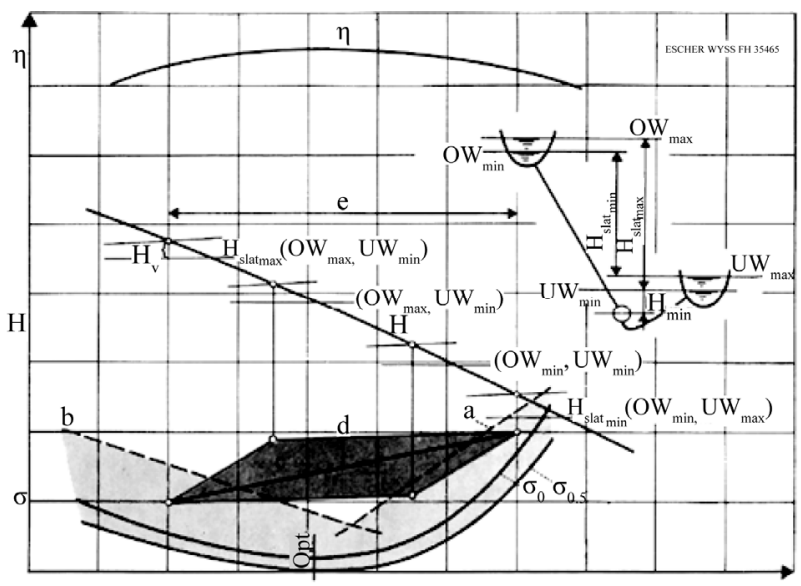

Figure 2. Operating range in pump mode.

the runner.

Drawing this consideration further, one can conclude that a pump-turbine is always too large to operate as a turbine. Best efficiencies of the turbine characteristics are outside the actual operating range of the machine (see Figure 3) - one of the drawbacks of a pump-turbine operating with fixed speed.

In order to define the limits of the operating range, suction and pressure side cavitation limits and the pump stability limit have to be known.

At larger heads cavitation starts at the suction side of the runner blade inlet in pump mode. As the velocity triangle changes towards lower heads, vapour pressure occurs at the pressure side of the blade in pump mode. An additional limitation occurs at the operation at large heads in pump mode due to instable flow phenomena in the hydraulic machine. Recirculation zones occur when the flow in pump mode minimizes and the pump pressure goes towards its maximum. Flow and power swings are the consequence which neither allows stable operation nor synchronization.

Especially for wide operating ranges-which often occur at pumped storage plants with lower heads-pressure and suction side cavitation substantially reduces the 


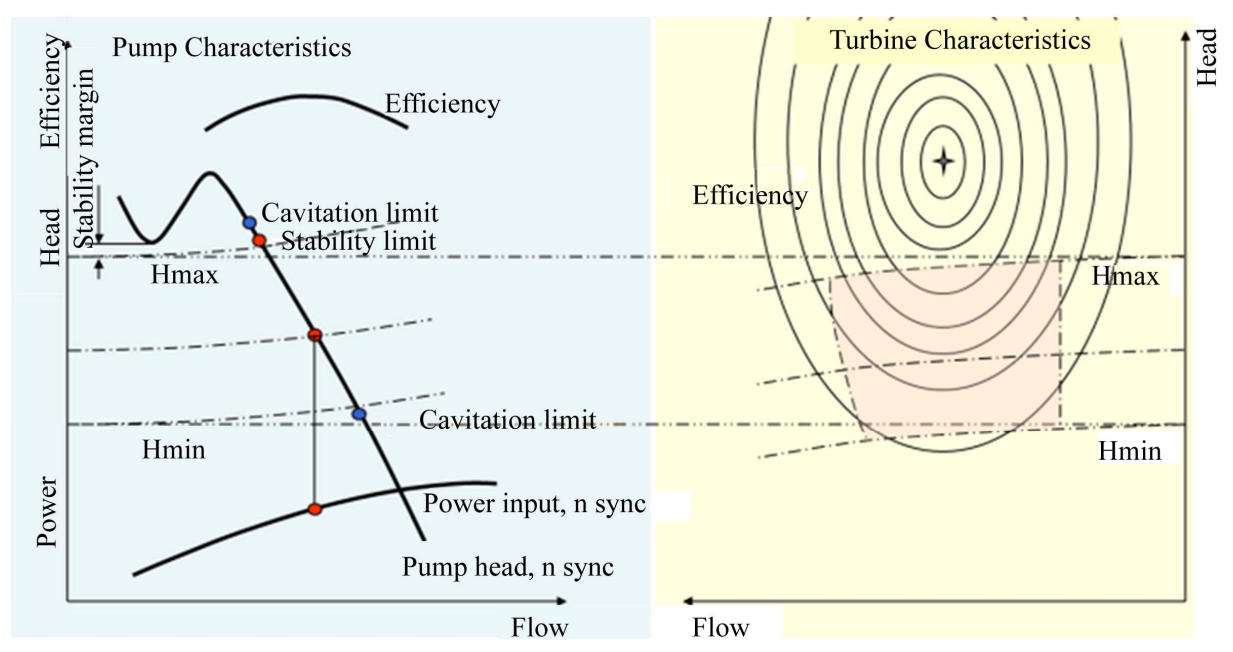

Figure 3. Pump-turbine characteristics for fixed speed operation.

usable range of application (see Figure 4, range No. 1). Large submergences are required to prevent cavitation abrasion. However, increasing submergences mean a low setting level of the machine which results in high civil work costs.

The above described cavitation effect occurs at extreme maximum and minimum head. At medium heads in the middle of the operating range (see Figure 4) the flow angle in pump mode corresponds much better to the geometrical blade angle. Therefore cavitation behaviour in this range is much better. However this advantage cannot be used in fixed speed operation.

In turbine mode the guide vane mechanism is used to regulate the machine, i.e. to change the flow and therefore power output. Regardless of the head the flow can be varied and the required power delivered to the grid can be controlled.

This property is not existent in pump mode. Changing the guide vane angle has very little effect on the power input. By closing the guide vanes the flow reduces but at the same time efficiency decreases. This leads to no significant change in power input. Consequently a pumpturbine with fixed speed cannot be regulated in pump mode by changing the guide vane angle. The plant operator has no possibility to control power. Depending on the pump characteristics the upper and lower reservoir level determines the flow which the machine pumps and therefore defines how much power is consumed from the grid. This unfavourable behaviour of the machine fits less and less to today's market requirements.

\subsection{Operation at Variable Speed}

Seeing the strong limitations of the operation at one single speed, immediately the question arises what happens when the speed is varied (see Figure 5). Above mentioned cavitation limitations can be side-stepped by sim-

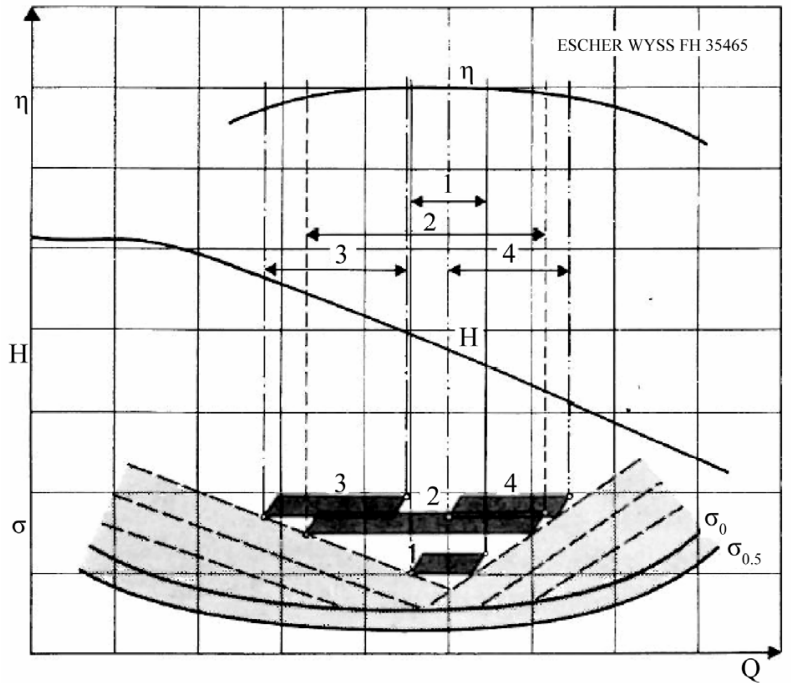

Figure 4. Influence of width of operating range on setting level.

ply changing the speed. At maximum head where the machine comes close to suction side cavitation the speed can be reduced to have pump inflow angles which better correspond to the geometrical runner blade angle. Vice versa at minimum head the speed can be increased to avoid pressure side cavitation and to extend the operating range to larger flow.

The machine can always be operated at best efficiency point in pump mode. With varying head the speed is adjusted to have flow angles which result in the highest possible efficiencies. Water resources are saved because less flow is required for the same pump input power.

As mentioned above one further advantage of the operation at best efficiency point is the improved cavitation behaviour. Less submergence is necessary which leads to reduced costs of the civil works which is a major part of the total costs of a new power plant. 


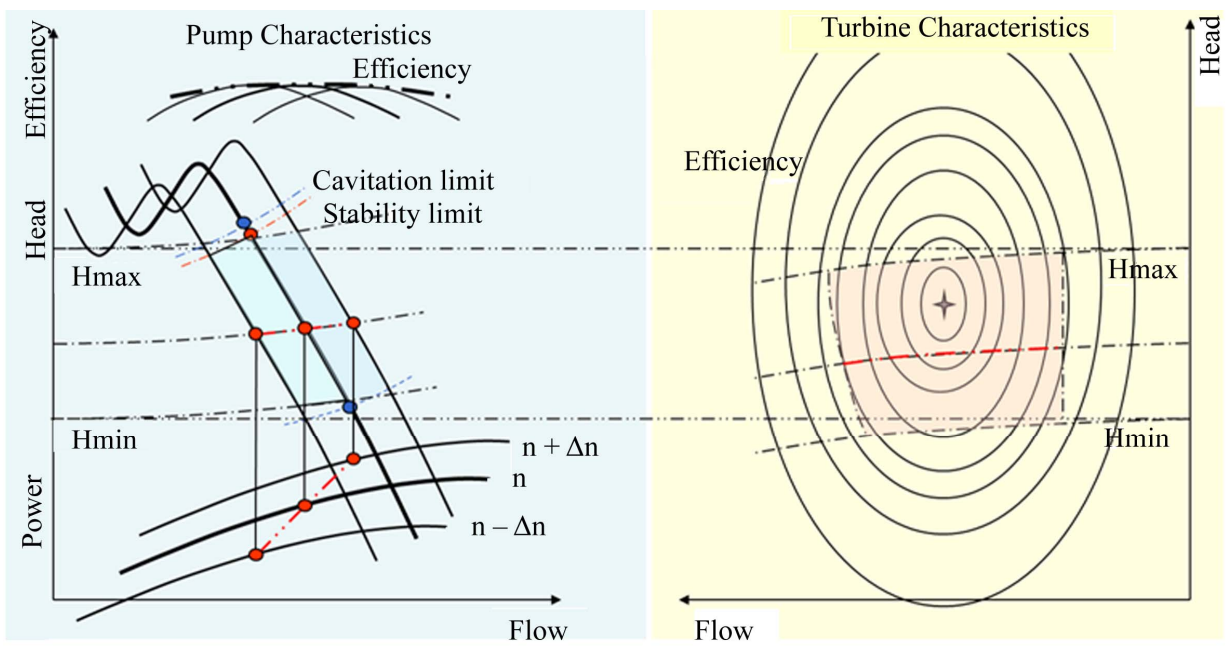

Figure 5. Pump-turbine characteristics for variable speed operation.

While in fixed speed operation pump input power is determined by the characteristics and the reservoir levels, the behaviour can be changed significantly with bringing in a new parameter. Speed can be changed which turns the before strongly limited machine into a regulated one. The machine becomes more flexible fulfilling the needs of today's energy markets. With varying speed the flow varies and consequently the pump input power varies while the pump net head stays more or less constant.

The already regulated machine with fixed speed in turbine mode becomes "double" regulated with variable speed which increases the flexibility even more. For fixed speed the runner diameter and the synchronous speed are too large to allow operation at best efficiency within the turbine operating range. The runner diameter is given and cannot be changed, but the speed can be reduced. The reduced speed shifts the whole turbine efficiency characteristics inside the operating range. In the whole operating range efficiencies are increased (see Figures 6 and 7). At medium and smaller heads and at part load this becomes much more pronounced with possible efficiency gains of the hydraulic machine of several per cent. On the other hand the converter losses will reduce the efficiency gain partly.

\subsection{Characteristics of the Concept}

In order to fulfil the needs of today's market requirements, ANDRITZ HYDRO has developed a new innovative concept (see Figure 8):

- Small decentralized pump storage plants with;

- Standardized pump turbines with variable speed;

- Synchronous motor-generator; and

- Full size converter.

The hydraulic pumped storage plant can be built locally, close to renewable generation like wind farms. This allows the increase of renewable energy without increasing the capacity of transmission lines.

Due to a full size converter, the pump input power can be varied continuously in a wide range, the possible head variation is large and the efficiency characteristic in pump and turbine mode is very flat over a wide operating range (see Figures 6 and 7).

Due to variable speed, the standardization comprises a few different types of machines only (see Figure 9). This results in a cost advantage compared to tailor-made small hydraulic pumped storage units.

\subsection{Targets in Developing the Concept}

\subsubsection{Hydraulic Models}

In order to keep costs low, the number of hydraulic models used in the concept had to be minimized. This saves engineering hours in the mechanical design because machines can be repeated. In this way the same type of machines can be used for various pumped storage applications.

\subsubsection{Machine Sizes}

Due to the small number of machine sizes in the concept, the probability to reuse an existing design is increased and so also costs are minimized.

Regarding the overall dimensions of the hydraulic machine, special care was taken to keep the machines as small as possible. Smaller machines contribute to lower manufacturing costs. In addition they require less space resulting in lower civil work costs.

\subsubsection{Setting Level}

In order to avoid cavitation at pump inlet, pump-turbines require a certain submergence. This means the hydraulic machine has to be installed below the level of the lower reservoir. Otherwise cavitation restrictions would make operation impossible. By well-selected hydraulic pump- 


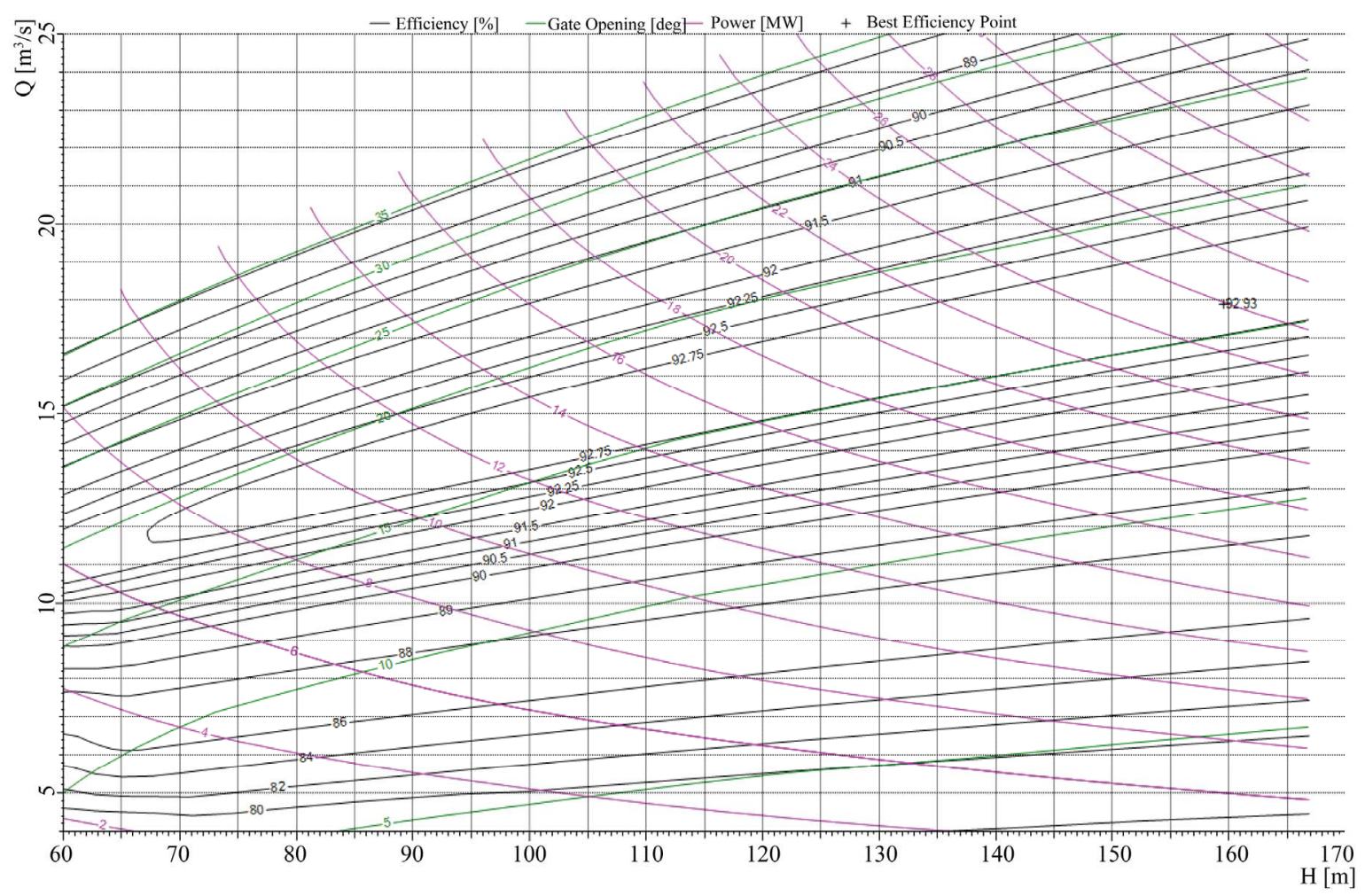

Figure 6. Turbine operation at variable speed: high efficiencies over wide range.

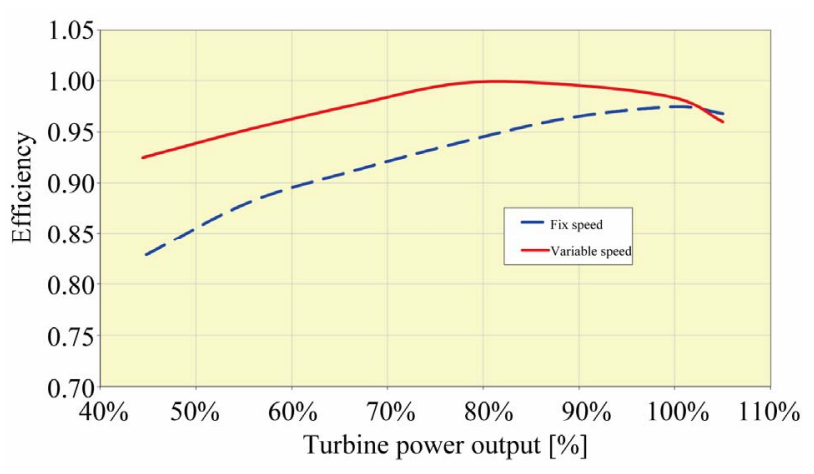

Figure 7. Turbine operation at variable speed: high efficiencies over wide range.

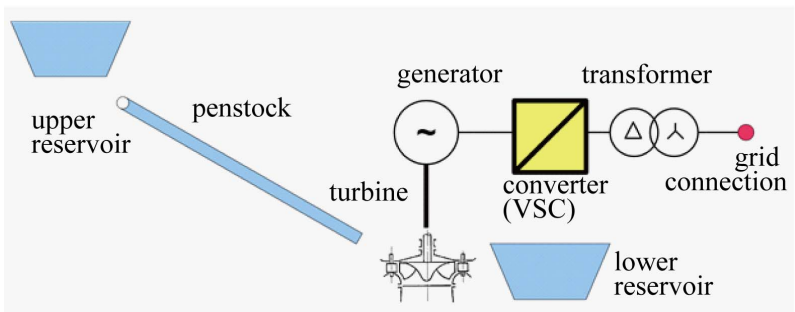

Figure 8. Electro-mechanical components of the ANDRITZ HYDRO concept.

turbine models, the submergence could be reduced to a minimum. This makes the new concept an even more economical solution.

\subsubsection{Design Adaptations}

The concept is based on well-proven pump-turbine designs [5,6] of ANDRITZ HYDRO for which model and site tests have been performed. Hydraulic design adaptations were performed to adapt to models to the new concept in order to keep costs low.

In the development of the concept, advantage has been taken of the widely used and long-term proven Compact Hydro series. Experience gained during many years in Compact Hydro business has been used to develop the new pump-turbine series.

\subsubsection{Standardization}

Standardized electro-mechanical components (including full size converter) are used to shorten delivery times (e.g. no model test are required) and to minimize costs.

\subsection{Application Range of New Concept}

The new Andritz Hydro concept was developed for heads up to $250 \mathrm{~m}$, flow rates of $40 \mathrm{~m}^{3} / \mathrm{s}$ and a maximum power output of $50 \mathrm{MW}$. With the concept both low head and high head pumped storage plants can be realized. The whole application range is covered with two hydraulic models with a total number of 17 machine sizes (see Figure 10).

The customer can select the appropriate model, for example PTM1700, which corresponds to his head varia- 


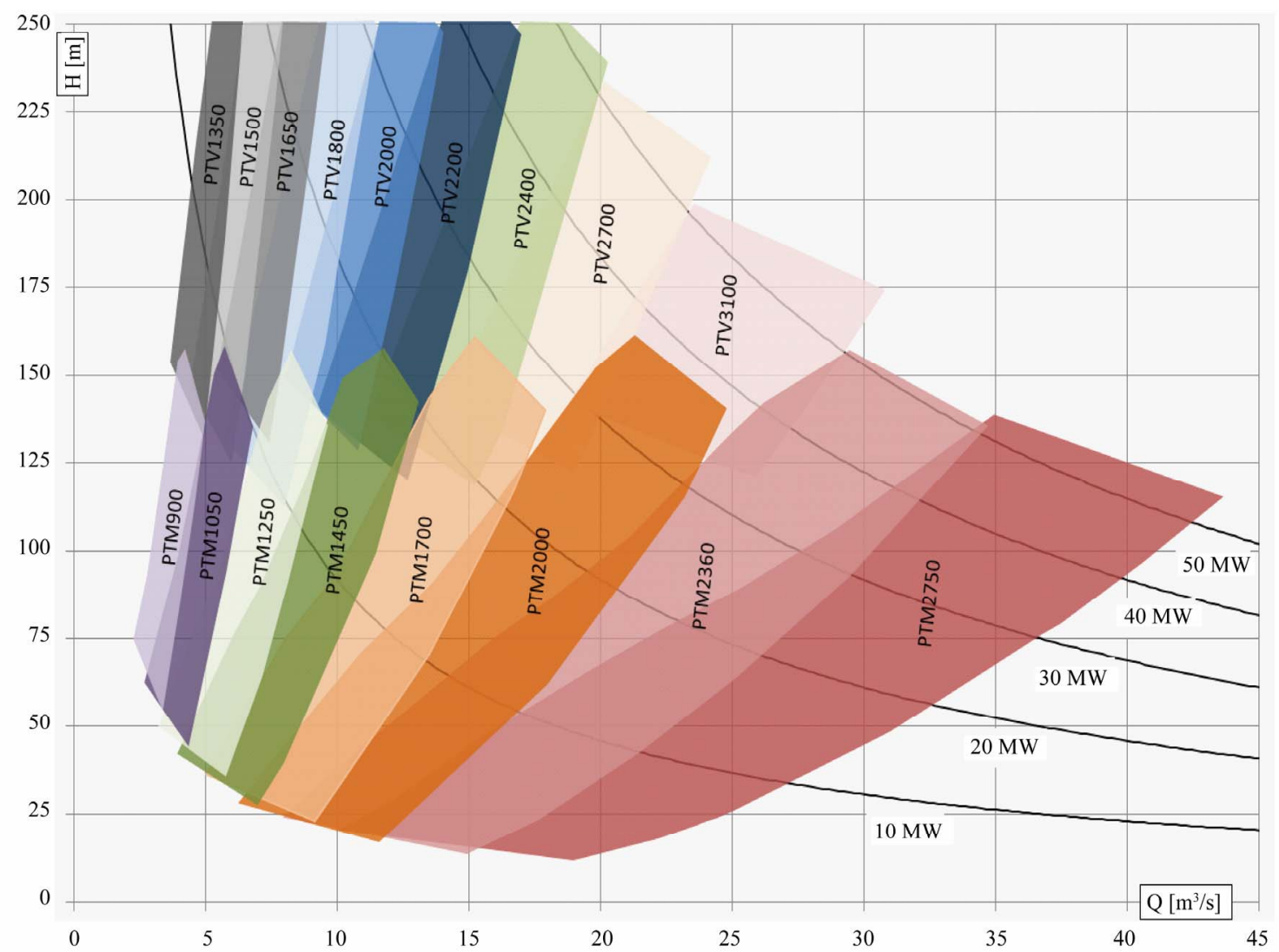

Figure 9. Application range of the new ANDRITZ HYDRO concept in pump mode.

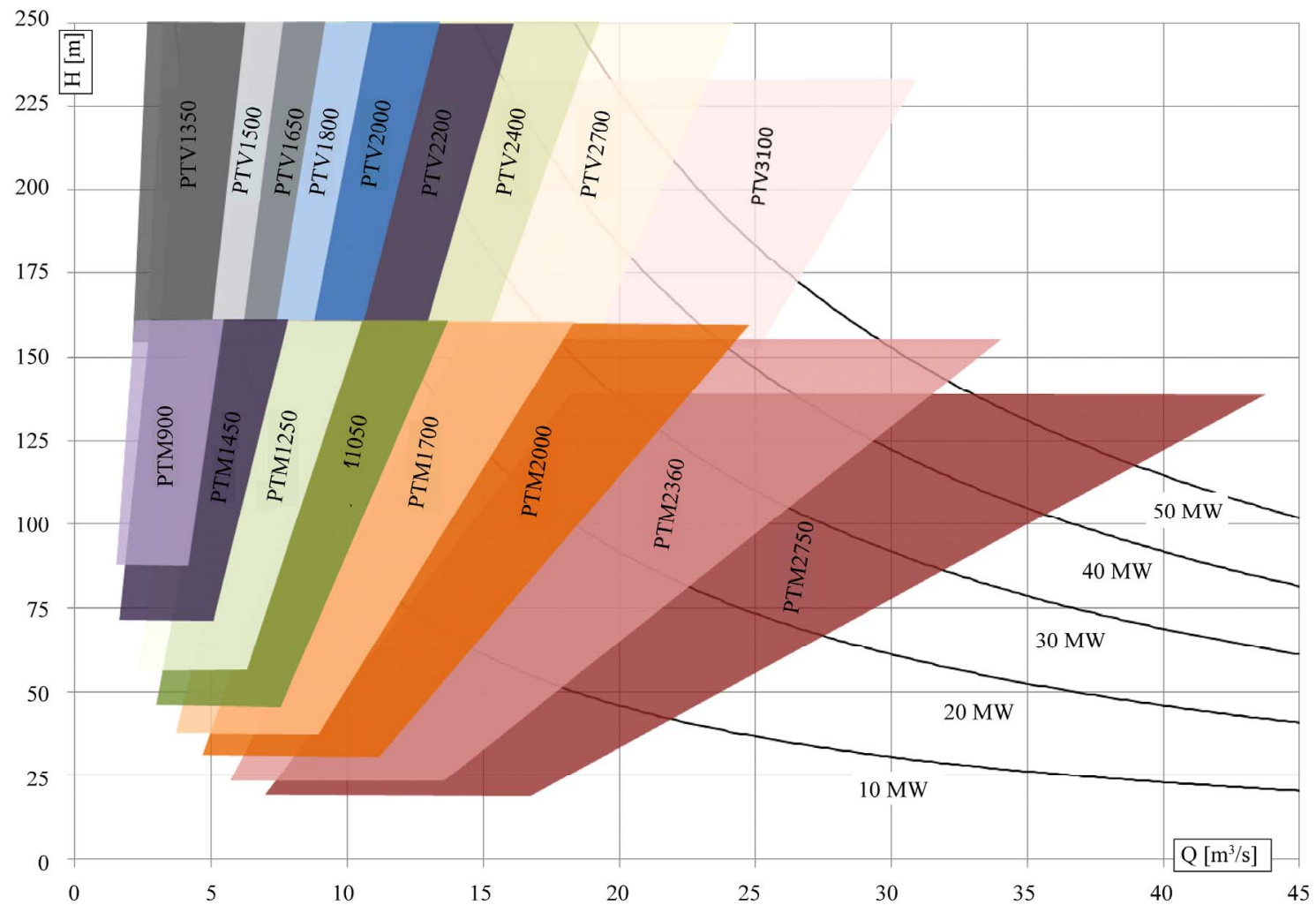

Figure 10. Application range of the new ANDRITZ HYDRO concept in turbine mode. 
tion and required power output. For example to balance for a $50 \mathrm{MW}$ wind park either two smaller machines PTM1700 or one larger machine PTM2360 can be selected.

\section{Benefits of the ANDRITZ HYDRO Concept}

The standardized pump-turbine concept improves return on investment for the customer. Due to its broad regulation flexibility combined with compact dimensions and low submergence requirements, it is a beneficial solution for which the energy market is asking for.

By using the concept's standardized pump-turbines, the utilisation of wind power can be increased in regions where otherwise the grid stability would not allow a higher percentage of installed wind power. The plant can be built locally, close to wind farms. The combination of a wind farm with a local hydraulic pumped storage plant allows the local utility to generate a much more constant and predictable amount of electricity. In this way the generation of renewable energy can be raised without the need to strongly increase the capacity of transmission lines.

In many regions small reservoirs or possibilities to create them with minimum effort already exist [7]. These reservoirs can serve as head and tail water of the plant. The high flexibility of the standardized pump-turbine offer new possibilities to find sites for building the power plant. The standardized pump-turbine enables operation with large variations in head and tail water, which is often a strong limitation of classical pumped-storage plants.
By selecting multi-purpose reservoirs (for storage of energy, storage of drinking water, irrigation, flood control, snow cannon storage etc.) the construction costs of the civil part are not fully charged to the power plant.

The ANDRITZ HYDRO concept provides electricity storage which is reliable, innovative and more economic than other storage technologies (batteries, etc.).

\section{REFERENCES}

[1] H. Keck, "Dezentrale Pumpspeicherung zur Lokalen Netzstabilisierung," OGE-Fachtagung Innsbruck, 2011.

[2] W. von Nessen Lapp, Vattenfall Europe Generation AG, P. Nowicki and VA TECH Escher Wyss, "Goldisthal: $4 \mathrm{x}$ 265 MW Pumpturbines in Germany,” 22nd IAHR Symposium, Stockholm, 2004.

[3] W. von Nessen Lapp, E. Dimter and Vattenfall Europe Generation AG, "Advantages of Variable Speed PumpTurbines,” 22nd IAHR Symposium, Stockholm, 2004.

[4] R. Schürhuber, J. Hell, A. Lechner and M. Pichler, “Optimized Pump Storage Concepts by Means of Converter Technology," The International Journal on Hydropower \& Dams, 2012

[5] M. Sallaberger, C. Gentner, C. Widmer, U. Henggeler and ANDRITZ HYDRO Switzerland, "Stability of Pump Turbines, Challenges in Their Design," Hydrovision, Louisville, 2012.

[6] M. Sallaberger, C. Gentner, ANDRITZ HYDRO Switzerland, "Challenges in Pump Turbine Development," Hydrovision, Prague, 2011.

[7] R. Lacal and E. Tzimas, "SETIS Expert Workshop on the Assessment of the Potential of Pumped Hydropower Storage,” Technical Report, Petten, 2012. 